\title{
Establishment of Network Platform of Virtuai Teaching Laboratories in Colleges and Universities Based on JSP Technology
}

\author{
Jun Luo and Wenxuan $\mathrm{Gu}^{\mathrm{b}}$ \\ ${ }^{a}$ College of Economic \& management. Wuhan Polytechnic University, China \\ ${ }^{b}$ International College. Wuhan Polytechnic University, China \\ a17572003@qq.com and b1319028827@qq.com
}

Keywords: JSP, virtual technology, network technology, distance education

\begin{abstract}
Virtual network laboratory system is the application of virtual reality technology to simulate the experimental software system. Based on the virtual network teaching platform using the JSP technology development, used to solve the current virtual education the existence of the problem. This paper introduced an overview of virtual teaching laboratory network platform, to create a virtual teaching laboratory platform with virtual technology and construction of platform strategies.
\end{abstract}

\section{Introduction}

With the rapid development of computer technology, multimedia technology and network technology, modern education technology crackles had been changed dynamically, more and more modern information technology experiment project and virtual laboratory have been established.

Virtualization is a type of technology that allows a single computer to do the same job that would normally be required of multiple computers (refer the Fig.1). It does this through sharing resources from one computer through a large number of environments.

Both virtual desktops as well as virtual servers will give you the ability to house more than one OS, and you can also host many applications locally and in locations which are remote. This frees you from the geographical and physical limitations that you would normally have to face.

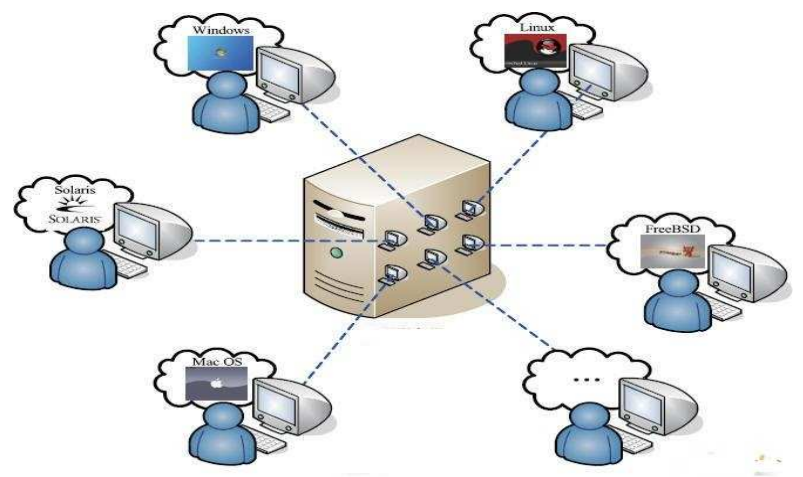

Fig. 1 The schematic diagram of virtual technolgy

Another advantage of virtualization is the savings you get in terms of energy. You also get greatly reduced capital costs because your hardware is used in a much more efficient way. It is also important to get high availability in terms of your resources, and you can also get enhanced desktop management, along with a much better level of security, and rapid disaster recovery methods. All this can be made available to you when you construct infrastructure which is virtual.

One of the most important aspects of virtualization is the virtual infrastructure. Virtualization allows for a number of opportunities which can be used to enhance both capital and the efficiency of operations. While safe partitioning has typically been used in the past, virtualization allows you to go far beyond these limitations. 
Virtual technology has now made it possible to make use of the power of virtualization in its full capacity. It can be done in environments that are production level, and this is accomplished through the construction of virtual management and automation abilities which are connected to a high end hypervisor.

Virtual network teaching platform is a new field of education based on virtual technology, which provided a real-time, regional restriction of network teaching environment, help to complete the design sex and exploring education. The research and application of virtual teaching, through the virtual teaching platform to students, teachers, and academic affairs administrator and the system administrator provide teaching management functions.

\section{Design of virtual teaching platform}

Virtual teaching platform is different from all kinds of virtual experiment, virtual laboratory and each network virtual laboratory, the goal is make it more extensive, and long-term perspective to solve of integration modern experimental teaching technology resources, share of the teaching resources. Virtual teaching platform is based on the Internet foundation, integrated design of virtual experiment, development tools and technology, all kinds of virtual experiment resources, and has the assessment and management function of large web.

Virtual teaching platform can be divided into five subsystems from the logical mode: student learning platform, teacher's online teaching system, resource center, teaching data analysis system, comprehensive evaluation system.

1. student learning platform

Establish website of support learning mode of the course autonomously. Expand course teaching space, autonomous learning website is different from the general teaching website; it can structure and expand to resources and procession of the learning, and provides discussion and evaluation in Internet. Establish website of support learning provides learning resources and realize of learning environment in collaboration way. Autonomous learning website including four aspects: professional knowledge, professional database, project cooperation and professional learning evaluation.

Professional knowledge including two parts: teaching synchronization and the link of learning resources, this aspect shows the related teaching information and local unique resources, provides to basic knowledge to learner.

Professional database is mainly about project knowledge resources, including the corresponding text, images, audio, video, etc.

Project cooperation is based on the bbs. It provides a learning communication platform to teachers and students. Here, you can explore problems which you are interested; also you can invite other learners to take part in, so as to create a project to explore and collaborative learning environment.

Professional learning evaluation include: practice of online learning evaluation, unit test, students' works, and personal learning situation analysis and others.

2. The construction of online teaching system

Online teaching system provides important supply based on classroom teaching way. It can extend the time and space of teacher teaching and standardize the process of teacher teaching. Standardization help reduce the difference of teaching level, the teaching experience of teachers teaching effect, the gap, students can get teaching level of the same basic teaching; And, it can provide necessary platform to online teaching system for teacher education. Online teaching system includes the following sections:

The online system of preparing lessons: teachers can design of the teaching system and process with online system of preparing lessons, it can generate individual teachers teaching calendar automatically, so planed the whole teaching process scientifically and reasonably. 
The online assignment management: teacher can design assignment management by the system include design operation of the chapter, layout and collection, students the assignments can be statistical automatically and generate reports through this system: students the assignments can be used as a phased evaluation indexes into the comprehensive assessment system.

Analysis of situation of class learning: teachers learn the study progress, scoring average of specific knowledge through analysis of situation of class learning. Understand difficult in course difficult in the process of teaching by using the results of analysis of the module.

Analysis of students learning situation: teachers can analysis the situation of specific student by using it. It includes mainly: percentage complete the assignments, the accuracy of finish the homework, the test score of the unit.

\section{The development of network support}

In recent years, the rapid development of Internet, encourage people to the understanding of the construction and concepts of the Internet. The situation of information and network were changed by progress of parallel computing, distributed computing, grid computing, and other computing technology. Network as a large technology service platform will support for the construction of virtual education platform.

Hardware support: We can realize the available virtualization of existence hardware resources through the virtualization software; further more realize the management, scheduling and application of virtual technology. The virtual platform based on virtualization hardware, can reduce the construction cost greatly, and also reduce maintenance costs in use procession.

Software support: With the development of Internet technology, software service as a application of software is gradually rise. This software service support construction of platform and operations of software lately. The first is to book special software used online service in construction and operation process of website, it can save a lot of money and manpower; the second is to secure the upgrade and maintenance of software, so provide reliable guarantee of the virtual platform.

\section{The development of virtual experiment teaching support}

Education information is the key of modern education; information technology is attractive in education field. The virtual experiment teaching includes three aspects as fellows:

Development tools level: With the development of the virtual experimental teaching, from it can be used in simple simulation instruments and to the advanced instruments simulation stage. For example, the Labview software, we can design the instrument based on needs of our experiment teaching, and the software has strong function of network, it can undertake web publishing with the built-in web Server, make the client do experiment with web browser.

Virtual experimental project level: In recent years, due to the need of teaching and research, the virtual experimental items of professional, academic is developed; many of the items have application values. These programs can satisfy to the needs of the web site in early experiment project stage.

Virtual laboratory level: With the rapid development of all kinds of simulation lab, virtual laboratory and network laboratory but also have sprung up come out, they provide a good foundations to virtual learning platform, especially those who have special hardware and software facilities in the network virtual laboratory support the establishment of virtual learning platform.

\section{Conclusion}

In summary, as the network education experimental teaching in virtual learning platform, it is an indispensable part of technology, and it can improve the quality of virtual learning, it also used in the virtual learning of network technology. Virtual network can solve the conflict between in the past mode of theoretical teaching network education and experimental teaching in the long distance, and realize network education completely. Virtual learning not only plays an effective role in remote 
education, but also provides new idea to other conventional education ways. Network and virtual learning technology realize the integrity of the request of the generation of virtual experiment and application of network education. And the key network of virtual laboratory resources construction is not completely hardware environment, it is depend on attention to the implementation of the measures and organization. With the generation of virtual learning platform, it will promote students initiative and enhancement creative ability by virtual learning platform, so as to improve the quality of network education.

\section{References}

[1]P. Barham et al., "Xen and the Art of Virtualization,"Proc. 19th ACM Symp. Operating Systems Princi-ples, ACM Press, 2003, pp. 164-177.

[2]Microsoft Corp., "Microsoft Virtual Server 2005 Technical Overview," 2004; http://download.microsoft.com/download/5/5/3/55321426-cb43-4672-9123-74ca3af6911d/VS2 005TechWP.doc.

[3] G.W. Dunlap et al., "ReVirt: Enabling Intrusion Analysis through Virtual-Machine Logging and Replay," Proc. 5th Symp. Operating Systems Design and Implementation, Usenix, 2002, pp. 211-224

[4] T. Garfinkel et al., "Terra: A Virtual Machine-Based Platform for Trusted Computing,” Proc. 19th ACM Symp. Operating Systems Principles, ACM Press,2003, pp. 193-206.

[5] P.B. England et al., “A Trusted Open Platform,”Computer, July 2003, pp. 55-62.

[6] VMware Inc., "Building Virtual Infrastructure withVMware VirtualCenter," white paper V00014-20001205, 2004; www.vmware.com/pdf/vi_wp.pdf.

[7] A. Whitaker, M. Shaw, and S. Gribble, "Scale and Performance in the Denali Isolation Kernel," Proc.5th Symp. Operating Systems Design and Implementation, Usenix, 2002, pp. 195-210.

[8] T.C. Bressoud and F.B. Schneider, "Hypervisor-Based Fault Tolerance," Proc. 15th ACM Symp. Operating Systems Principles, ACM Press, 1995, pp. 1-11.

[9] Intel Corp., "Intel Virtualization Technology Specification for the IA-32 Architecture;" www.intel.com/technology/vt/.

[10]Intel Corp., "Intel Virtualization Technology Specification for the Intel Itanium Architecture;" www.intel.com/technology/vt/. 